\title{
Effects of dexamethasone coadministered with oseltamivir on the pharmacokinetics of oseltamivir in healthy volunteers
}

This article was published in the following Dove Press journal:

Drug Design, Development and Therapy

9 March 2017

Number of times this article has been viewed

\author{
Kyungho Jang ${ }^{1,2, *}$ \\ Min-Kyoung Kim ${ }^{3,4, *}$ \\ Jaeseong $\mathrm{Oh}^{\prime}$ \\ SeungHwan Lee' \\ Joo-Youn Cho' \\ Kyung-Sang Yu' \\ Tai Kiu Choi ${ }^{3}$ \\ Sang-Hyuk Lee ${ }^{3,4}$ \\ Kyoung Soo $\mathrm{Lim}^{4}$
}

'Department of Clinical Pharmacology and Therapeutics, Seoul National University College of Medicine and Hospital, Seoul, ${ }^{2}$ Center for Clinical Pharmacology and Biomedical Research Institute, Chonbuk National University Medical School, Jeonju, ${ }^{3}$ Department of Psychiatry, ${ }^{4}$ Department of Clinical Pharmacology and Therapeutics, CHA University School of Medicine and CHA Bundang Medical Center, Seongnam, Republic of Korea

*These authors contributed equally to this work

\begin{abstract}
Purpose: Oseltamivir is widely used in the treatment and prophylaxis of influenza A and B viral infections. It is ingested as an oral prodrug that is rapidly metabolized by carboxylesterase 1 (CES1) to its active form, oseltamivir carboxylate. Dexamethasone is also used in the treatment of acute respiratory distress syndrome, a severe complication of influenza; however, its influence on the pharmacokinetics (PK) of oseltamivir is controversial. The aim of this study was to investigate the effects of coadministering oseltamivir and dexamethasone on the PK of oseltamivir in healthy volunteers.
\end{abstract}

Methods: An open-label, two-period, one-sequence, multiple-dose study was conducted in 19 healthy male volunteers. Oseltamivir $(75 \mathrm{mg}$ ) was orally administered on Day 1 and Day 8 , and dexamethasone $(1.5 \mathrm{mg}$ ) was administered once daily from Day 3 to Day 8 . Serial blood and urine samples were collected for PK analysis of oseltamivir and oseltamivir carboxylate on Day 1 and Day 8. Oseltamivir and oseltamivir carboxylate concentrations in plasma and urine were determined using liquid chromatography-tandem mass spectrometry.

Results: Area under the plasma concentration-time curve (AUC) of oseltamivir and oseltamivir carboxylate decreased after dexamethasone treatment for 6 days. The geometric mean ratio ( $90 \%$ confidence interval) of the metabolic ratio (oseltamivir carboxylate $\mathrm{AUC}_{0-48 \mathrm{~h}} /$ oseltamivir $\mathrm{AUC}_{0-48 \mathrm{~h}}$ ) was $0.92(0.87-0.97)$. The amount of unchanged oseltamivir excreted in urine increased by $14 \%$ after dexamethasone treatments.

Conclusion: Coadministration of dexamethasone with oseltamivir slightly decreased systemic exposure to oseltamivir and oseltamivir carboxylate in healthy volunteers. This result suggests that CES1 is inhibited by dexamethasone in humans. However, coadministration of oseltamivir and dexamethasone did not appear to have a clinically relevant effect on the PK of oseltamivir; based on these results, dexamethasone can be coadministered with oseltamivir.

Keywords: carboxylesterase, steroid, influenza, ARDS

\section{Introduction}

Carboxylesterase (CES) plays an important role in the hydrolysis of various drugs such as angiotensin-converting enzyme inhibitors, statins, central nervous system stimulants, immunosuppressants, and antiviral agents. ${ }^{1,2} \mathrm{CES}$ activity is a major determinant of the pharmacokinetics (PK) and pharmacodynamics (PD) of these drugs; ${ }^{3}$ thus, modulation of CES activity may alter drug metabolism and PK, which may lead to drug toxicity or improve efficacy. In particular, drug-drug interactions can affect the activity of CES, significantly impacting the therapeutic effects of substrate drugs. ${ }^{4}$ For example, ethanol inhibits the hydrolysis of methylphenidate, a CES1 substrate, and increases the maximum concentration $\left(C_{\max }\right)$ and area under the concentration-time curve (AUC) of
Correspondence: Kyoung Soo Lim Department of Clinical Pharmacology and Therapeutics, CHA University School of Medicine and CHA Bundang Medical Center, 59 Yatap-ro, Bundang-gu, Seongnam 463-7/2, Republic of Korea Tel +82317805324

Fax +82317805305

Email dr.kyoungsoo.lim@gmail.com (c) (1) (5) 2017 jang et al. This work is published and licensed by Dove Medical Press Limited. The full terms of this license are available at https:///www.dovepress.com/terms.php

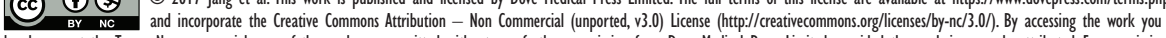
hereby accept the Terms. Non-commercial uses of the work are permitted without any further permission from Dove Medical Press Limited, provided the work is properly attributed. For permission for commercial use of this work, please see paragraphs 4.2 and 5 of our Terms (https://www.dovepress.com/terms.php). 
methylphenidate. ${ }^{4,5}$ Therefore, alternatives to methylphenidate treatment may be considered in patients with attentiondeficit hyperactivity disorder and alcohol abuse. ${ }^{5}$

Oseltamivir, a CES substrate, is widely used in the treatment and prophylaxis of influenza A and B viral infections. ${ }^{6}$ Oseltamivir is readily absorbed from the gastrointestinal tract after oral administration and is predominantly converted by CES1 to its active metabolite, oseltamivir carboxylate, which is an inhibitor of influenza virus neuraminidase. At least $75 \%$ of an oral dose of oseltamivir enters the systemic circulation as oseltamivir carboxylate. ${ }^{7,8}$ Renal elimination of oseltamivir carboxylate accounts for more than $99 \%$ of the administered dose..$^{7-9}$ Generally, the recommended dose for therapeutic treatment in adults with normal renal function is $75 \mathrm{mg}$ twice a day for 5 days. ${ }^{10}$ Multiple agents are combined to treat the symptoms of influenza patients or to gain synergistic effects. Information derived from previous pharmacology and PK studies of oseltamivir suggests that clinically significant drug interactions are unlikely. ${ }^{9}$

Dexamethasone, a type of steroid medication, is used to treat a wide variety of inflammatory and autoimmune diseases. Especially, steroids may have beneficial effects in acute respiratory distress syndrome (ARDS), a severe complication of influenza, which is controversial. ${ }^{11}$ It has been documented that dexamethasone is a moderate inducer of cytochrome P450 3A4; however, the influence of dexamethasone on CES is controversial. Previously, Takahashi et $\mathrm{al}^{12}$ in 2009 demonstrated that dexamethasone weakly inhibits the formation of imidaprilat from imadipril by CES1 in human liver, whereas a study by Zhu et $\mathrm{al}^{13}$ demonstrated that exposure of cultured human hepatocytes to dexamethasone causes a slight increase in human CES1 and CES2, two major forms of human liver microsomal CES.

In summary, drug interactions between oseltamivir and dexamethasone can occur through the inhibition or induction of CES, which could change the PK and PD of oseltamivir. Conflicting results have been obtained in studies examining the influence of dexamethasone on CES activity; however, drug interaction data for oseltamivir and dexamethasone in humans are insufficient. Therefore, efforts are needed to determine the potential for drug interactions between oseltamivir and dexamethasone in humans as well as the underlying mechanisms. The aim of this study was to evaluate the impact of coadministering dexamethasone and oseltamivir on the PK of oseltamivir in healthy volunteers.

\section{Materials and methods}

This study was conducted at the Clinical Trials Center (CTC), CHA Bundang Medical Center, Seongnam, Republic of Korea, in compliance with the ethical principles of the Declaration of Helsinki, International Conference on Harmonization Good Clinical Practice Guideline, and local laws and regulations. The protocol was approved by the Institutional Review Board of CHA Bundang Medical Center and registered at National Research Institute of Health (CRIS: KCT0001533). All the subjects provided written informed consent after a detailed explanation of the study prior to any study procedure.

\section{Subjects}

Males aged 20-45 years with a body mass index of $19-27 \mathrm{~kg} / \mathrm{m}^{2}$ and who were in good general health based on a detailed medical history, physical examination, vital signs, electrocardiography (ECG), and clinical laboratory evaluations (including hematology, liver function tests, renal function tests, blood glucose, urinalysis [including urine drug screening], and seroimmunology [hepatitis B surface antigen, anti-hepatitis $\mathrm{C}$ virus antibody, and anti-HIV antibody]) were included in this study. Subjects were excluded if they had the following: a history of significant gastrointestinal, hepatic, renal, respiratory, cardiovascular, metabolic, immunological, or hormonal disorders; a history of drug or food allergies; taken any prescription medication within 2 weeks prior to the first administration of the study drug; followed a diet that would affect the absorption, distribution, metabolism, and elimination of the drugs; a positive drug or alcohol screening; smoked 10 or more cigarettes per day within 3 months; or participated in a clinical trial during the last 3 months prior to the start of the study.

\section{Study design}

An open-label, two-period, single-sequence study was performed (Figure 1). Twenty healthy Korean male subjects were enrolled. All subjects were admitted to the CTC at CHA Bundang Medical Center on the day before oseltamivir administration in Period 1. On the following day (Day 1) after overnight fasting, a single dose of $75 \mathrm{mg}$ oseltamivir (Tamiflu ${ }^{\circledR}$ Capsule; Roche Registration Ltd., Welwyn Garden City, UK) was orally administered with $240 \mathrm{~mL}$ of water, and serial blood and urine samples for PK evaluation were taken over $48 \mathrm{~h}$. Daily doses of dexamethasone $(0.5 \mathrm{mg}$, three tablets) were administered orally for 6 days every morning from Day 3 to Day 8. On Day 8, oseltamivir and dexamethasone were coadministered orally at the same time in the morning, and subjects underwent the same procedure as they did in Period 1 (Figure 1).

For PK analysis of oseltamivir and oseltamivir carboxylate, blood samples were collected at 0 (ie, pre-dose), 0.5, 1, 1.5, $2,3,4,5,6,8,10,12,24$, and 48 h post-dose; urine samples 


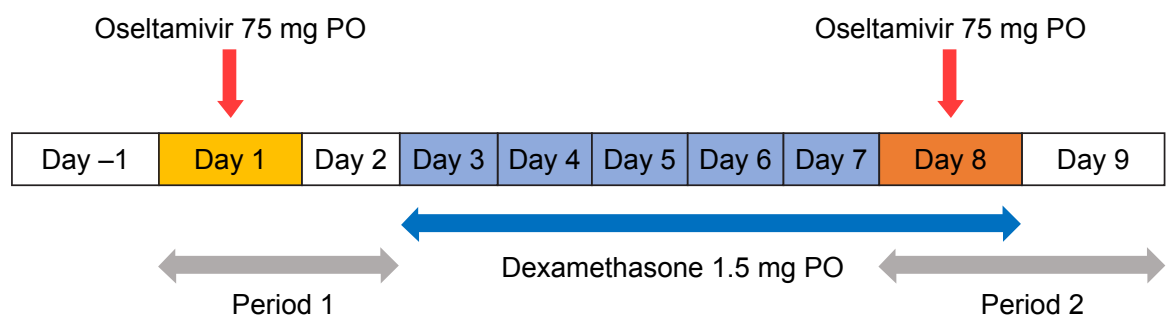

Figure I Study design.

Notes: Oseltamivir 75 mg one capsule (Tamiflu ${ }^{\circledR}$; Roche Registration Ltd., Welwyn Garden City, UK) and dexamethasone 0.5 mg three tablets (YuhanMedica, Cheongju, Republic of Korea) were used.

Abbreviation: PO, per os.

were collected up to $24 \mathrm{~h}$ post-dose. Tolerability was evaluated throughout the entire study period by examining the incidence and type of adverse events (AEs), as well as changes in clinical laboratory test values, physical examinations, vital signs, and 12-lead ECGs.

\section{Determination of oseltamivir and oseltamivir carboxylate concentration}

Oseltamivir and oseltamivir carboxylate concentrations in plasma and urine were determined using a highly specific and sensitive method of liquid chromatography-tandem mass spectrometry (Agilent 6490 Triple Quadrupole; Agilent Technologies, Santa Clara, CA, USA). To prepare the samples for analysis, an aliquot of the plasma or urine specimen was mixed with acetonitrile in the presence or absence of oseltamivir carboxylate-d3, which was used as an internal standard. The mixture was vortexed for $30 \mathrm{~s}$ and then centrifuged for $10 \mathrm{~min}$ at 14,000 rpm. An aliquot of the supernatant was transferred to an autosampler vial, and $2 \mu \mathrm{L}$ was injected onto a Kinetex HILIC column $(50 \times 2.1 \mathrm{~mm}, 5 \mu \mathrm{m}$; Phenomenex, Torrance, CA, USA) with a $3 \mathrm{~min}$ run at a flow rate of $0.3 \mathrm{~mL} / \mathrm{min}$ using gradient elution. Mobile phase A consisted of $10 \mathrm{mM}$ ammonium acetate in water, and mobile phase B consisted of $100 \%$ acetonitrile. Oseltamivir and oseltamivir carboxylate were quantitatively detected in the positive ionization of triple-quadrupole mass spectrometry equipped with electrospray ionization. The method was validated with a range of 0.5-100 and 20-20,000 ng/mL for oseltamivir in plasma and urine, respectively, and 2-500 and 500-100,000 ng/mL for oseltamivir carboxylate in plasma and urine, respectively.

\section{PK data analysis}

Plasma concentrations of oseltamivir and oseltamivir carboxylate were analyzed by noncompartmental analysis using Phoenix ${ }^{\circledR}$ WinNonlin ${ }^{\circledR}$ software version 1.3 (Certara, Princeton, NJ, USA). AUC for oseltamivir and oseltamivir carboxylate from time 0 to $48 \mathrm{~h}$ post-dose $\left(\mathrm{AUC}_{0-48 \mathrm{~h}}\right)$ was calculated using the linear-up, log-down trapezoidal method.
In addition, $\mathrm{AUC}$ from time zero to infinite time $\left(\mathrm{AUC}_{\text {inf }}\right)$ was calculated as the sum of $\mathrm{AUC}_{0-48 \mathrm{~h}}$ and the last quantifiable concentration divided by the slope of the final decline portion of the individual log-linear concentration-time curve. The metabolic ratio was calculated as oseltamivir carboxylate $\mathrm{AUC}_{0-48 \mathrm{~h}}$ /oseltamivir $\mathrm{AUC}_{0-48 \mathrm{~h}}$. The observed concentrations and times were used to estimate the $C_{\max }$ and time to reach the $C_{\max }\left(T_{\max }\right)$ for oseltamivir and oseltamivir carboxylate. The apparent terminal elimination rate constant $\left(\lambda_{z}\right)$ was estimated from a regression of log-transformed plasma concentrations of oseltamivir and oseltamivir carboxylate versus time over the terminal log-linear disposition portion of the concentration-time profiles. The elimination half-life $\left(t_{1 / 2}\right)$ was calculated as the natural logarithm of 2 divided by $\lambda_{z}$. Total apparent clearance (CL/F) of oseltamivir was calculated as the administered dose $\left(75 \mathrm{mg}\right.$ ) over the $\mathrm{AUC}_{0-48 \mathrm{~h}}$ (oseltamivir), and the apparent volume of distribution was calculated as the CL/F divided by $\lambda_{z}$. Total urinary excreted amount of oseltamivir and oseltamivir carboxylate during $24 \mathrm{~h}\left(\mathrm{Ae}_{0-24 \mathrm{~h}}\right)$ was calculated by multiplying the volume of excreted urine and urinary concentration of oseltamivir and oseltamivir carboxylate, respectively.

\section{Statistical analysis}

All the demographic characteristics and PK parameters are presented as arithmetic mean values and standard deviation (SD). A general linear model was developed to estimate the geometric mean ratios (GMRs) of the PK parameters and their $90 \%$ confidence intervals for oseltamivir and its metabolite (oseltamivir carboxylate). Differences were considered statistically significant when $P$-values were less than 0.05 . Statistical analyses were performed using SAS software version 9.3 (SAS Institute Inc., Cary, NC, USA).

\section{Results Study population}

A total of 20 healthy subjects were enrolled, 19 (95\%) of whom completed the study. One subject withdrew consent 

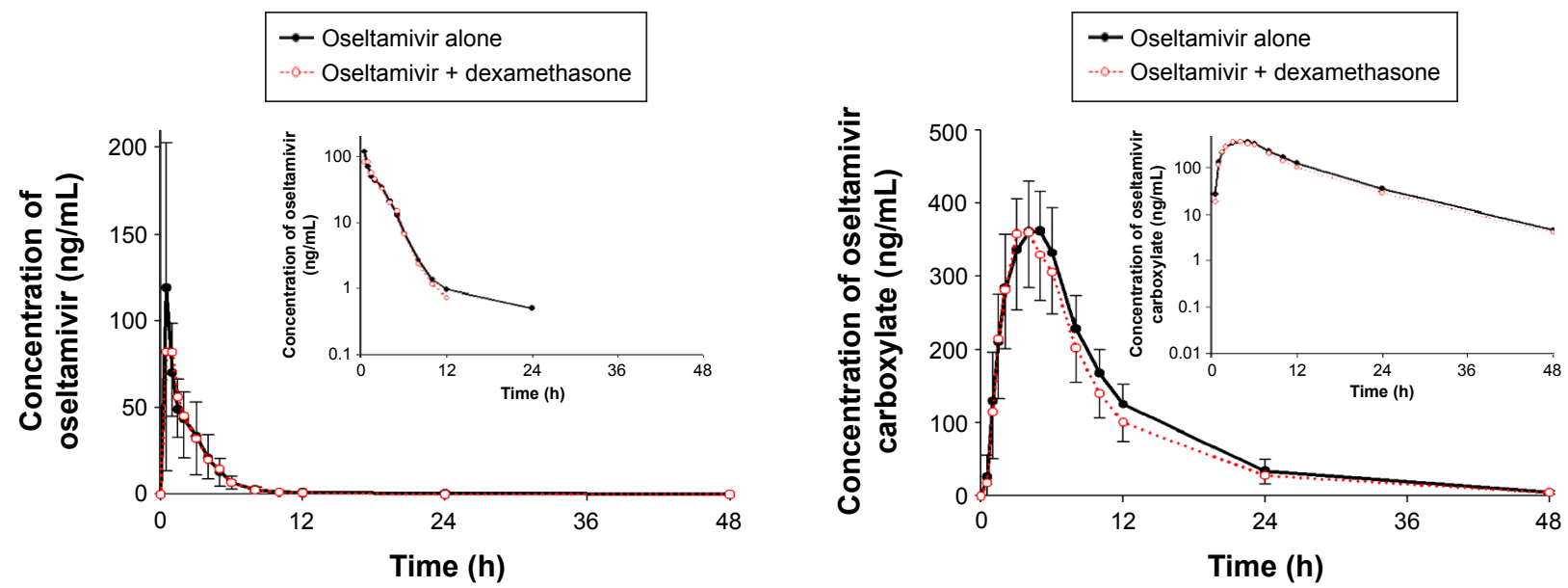

Figure 2 Mean plasma concentration-time profiles of oseltamivir (left) and oseltamivir carboxylate (right) after a single oral administration of oseltamivir (75 mg) and a single oral administration of oseltamivir $(75 \mathrm{mg})$ after 6 days of dexamethasone ( $1.5 \mathrm{mg})$ treatments (inset: log-transformed). The error bars represent the standard deviation.

prior to administration of dexamethasone on Day 3. The mean $\pm \mathrm{SD}$ of age, body weight, and body mass index were $27.7 \pm 3.8$ years, $70.7 \pm 6.7 \mathrm{~kg}$, and $23.0 \pm 1.7 \mathrm{~kg} / \mathrm{m}^{2}$, respectively.

\section{Effects of dexamethasone on oseltamivir PK}

The concentration-time profiles for oseltamivir before dexamethasone treatments were comparable to those after 6 days of dexamethasone administrations (Figure 2). Furthermore, the systemic exposure to oseltamivir in subjects coadministered with dexamethasone, based on $\mathrm{AUC}_{0-48 \mathrm{~h}}$ and $\mathrm{AUC}_{\text {inf }}$, was slightly lower (4\% and $5 \%)$ than that of subjects who were not administered dexamethasone, although this difference was not statistically significant (Table 1). In contrast, $\mathrm{AUC}_{0-48 \mathrm{~h}}$ and $\mathrm{AUC}_{\text {inf }}$ of oseltamivir carboxylate were approximately $12 \%$ and $11 \%$ lower, respectively, in subjects receiving multiple doses of dexamethasone than those administered oseltamivir alone, which were statistically significant outcomes (Table 1). The $C_{\max }$ of oseltamivir carboxylate after

Table I Pharmacokinetic parameters of oseltamivir and oseltamivir carboxylate after a single dose of oseltamivir (75 mg) orally administered and a single dose of oseltamivir $(75 \mathrm{mg})$ coadministered with dexamethasone after 6 days of dexamethasone (I.5 mg) treatment

\begin{tabular}{|c|c|c|c|c|c|c|c|c|}
\hline \multirow[t]{2}{*}{ PK parameters } & \multicolumn{2}{|l|}{ Oseltamivir } & \multirow{2}{*}{$\begin{array}{l}\mathbf{G M R}^{\mathrm{a}} \\
(90 \% \mathrm{CI})\end{array}$} & \multirow[t]{2}{*}{$P$-value } & \multicolumn{2}{|c|}{ Oseltamivir carboxylate } & \multirow{2}{*}{$\begin{array}{l}\text { GMR }^{a} \\
(90 \% \mathrm{CI})\end{array}$} & \multirow[t]{2}{*}{$P$-value } \\
\hline & $\begin{array}{l}\text { Oseltamivir } \\
\text { alone } \\
(n=19)\end{array}$ & $\begin{array}{l}\text { Oseltamivir }+ \\
\text { dexamethasone } \\
(n=19)\end{array}$ & & & $\begin{array}{l}\text { Oseltamivir } \\
\text { alone } \\
(n=19)\end{array}$ & $\begin{array}{l}\text { Oseltamivir }+ \\
\text { dexamethasone } \\
(n=19)\end{array}$ & & \\
\hline \multicolumn{9}{|l|}{$\overline{T_{\max }(\mathrm{h})}$} \\
\hline Median & 0.5 & I & & & 4 & 4 & & \\
\hline Range & $0.5-3.0$ & $0.5-4.0$ & & & $2.0-6.0$ & $2.0-5.0$ & & \\
\hline \multicolumn{9}{|l|}{$C_{\text {max }}(\mathrm{ng} / \mathrm{mL})$} \\
\hline Mean & $|3|$ & 117.1 & $0.92(0.75-1.12)$ & 0.47 & 380.34 & 381.15 & $0.99(0.94-1.04)$ & 0.71 \\
\hline SD & 72.63 & 53.69 & & & 57.73 & 89.48 & & \\
\hline \multicolumn{9}{|l|}{$\mathrm{AUC}_{0-48 \mathrm{~h}}(\mathrm{~h} \cdot \mathrm{ng} / \mathrm{mL})$} \\
\hline Mean & 230.15 & 219.02 & $0.96(0.90-1.02)$ & 0.21 & $4,022.46$ & $3,554.85$ & $0.88(0.85-0.91)$ & $<0.0001$ \\
\hline SD & 58.62 & 49.91 & & & 709.32 & 712 & & \\
\hline \multicolumn{9}{|l|}{$\mathrm{AUC}_{\mathrm{nf}}(\mathrm{h} \cdot \mathrm{ng} / \mathrm{mL})$} \\
\hline Mean & 232.97 & 221.2 & $0.95(0.90-1.01)$ & 0.19 & $4,083.08$ & $3,639.18$ & $0.89(0.87-0.91)$ & $<0.000$ I \\
\hline SD & 58.92 & 50.13 & & & 709.68 & 703.65 & & \\
\hline \multicolumn{9}{|l|}{$\mathrm{Ae}_{0-24 \mathrm{~h}}(\mathrm{mg})$} \\
\hline Mean & 2.44 & 2.68 & $1.14(1.01-1.30)$ & 0.08 & 42.85 & 47.18 & I.II (0.99-I.24) & 0.14 \\
\hline SD & 0.86 & 0.62 & & & 9.47 & 9.75 & & \\
\hline \multicolumn{9}{|l|}{ CLr (L/h) } \\
\hline Mean & 11.06 & 12.38 & I.2(I.02-I.4I) & 0.07 & 11.94 & 14.68 & $1.24(1.10-1.39)$ & 0.005 \\
\hline SD & 4.41 & 2.84 & & & 3.73 & 3.91 & & \\
\hline
\end{tabular}

Notes: a Geometric mean ratio $(90 \% \mathrm{Cl})$ of (oseltamivir + dexamethasone)/(oseltamivir alone). ${ }^{\ddagger} P$-value was measured using a mixed-effect model in SAS software version 9.3 . Abbreviations: PK, pharmacokinetics; GMR, geometric mean ratio; $\mathrm{Cl}$, confidence interval; SD, standard deviation; $T_{\max }$, time to maximum concentration; $C_{\max }$, maximum plasma concentration; AUC, area under the plasma concentration-time curve; Ae, amount of urine excreted; CLr, renal clearance. 

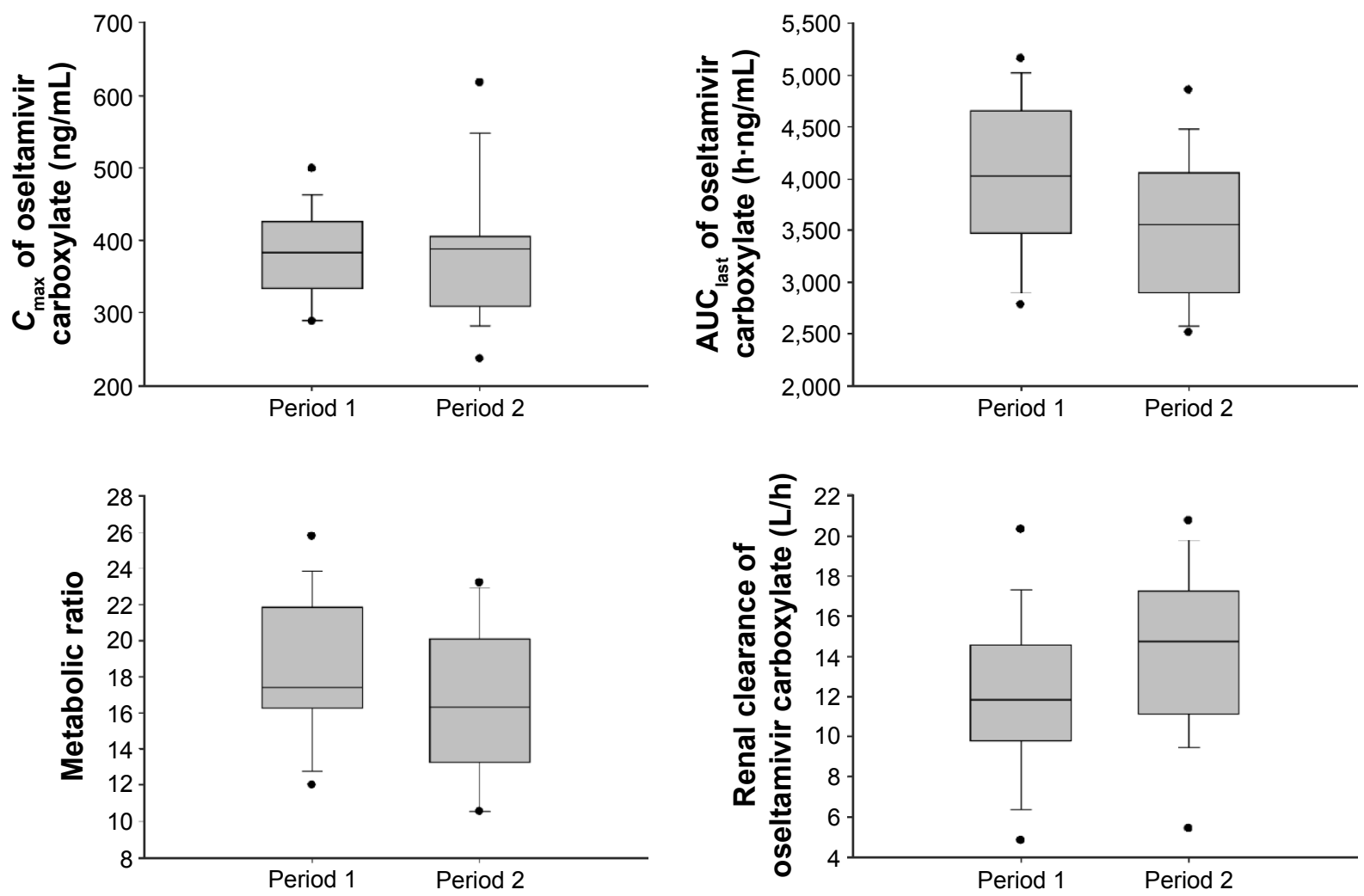

Figure 3 Comparison of PK parameters of oseltamivir and oseltamivir carboxylate with and without dexamethasone.. The metabolic ratio was calculated as $\mathrm{AUC}_{0-48}$ (oseltamivir carboxylate)/AUC ${ }_{0-48 \mathrm{~h}}$ (oseltamivir). The solid lines across the box, the top edge, and the bottom edge represent the median, the 75 th percentile, and the 25 th percentile, respectively. The horizontal lines connected with the whiskers extending from the box denote the 90th and I0th percentiles, respectively. Dots outside of the whiskers represent outliers.

Abbreviations: PK, pharmacokinetics; AUC, area under the plasma concentration-time curve.

treatments with dexamethasone was comparable to the $C_{\max }$ observed in subjects administered oseltamivir alone (Table 1 and Figure 3).

The metabolic ratio of oseltamivir was approximately $8 \%$ lower when coadministered with dexamethasone than when administered alone, which was statistically significant (Table 2 and Figure 3). The amount of oseltamivir and oseltamivir carboxylate excreted in the urine was greater after treatment with dexamethasone, and the GMR for the $\mathrm{Ae}_{0-24 \mathrm{~h}}$ of oseltamivir and oseltamivir carboxylate was 1.14 and 1.11, respectively (Table 1 and Figure 3).

\section{Tolerability}

No serious AEs occurred in this study, and unexpected AEs that could have influenced the outcome of the study were not reported. Vital signs of the study subjects, including blood pressure, pulse rate, body temperature, and the physical examination results, showed no clinically significant changes.

\section{Discussion}

Oseltamivir is extensively converted to its active metabolite by CES1. Dexamethasone is known to modulate the

Table 2 Metabolic ratio of oseltamivir after a single oral administration of oseltamivir $(75 \mathrm{mg})$ and a single oral administration of oseltamivir $(75 \mathrm{mg})$ after 6 days of dexamethasone $(1.5 \mathrm{mg})$ treatment

\begin{tabular}{|c|c|c|c|c|}
\hline \multirow[t]{2}{*}{ Parameters } & \multicolumn{2}{|c|}{ Oseltamivir carboxylate/oseltamivir } & \multirow[t]{2}{*}{$\mathrm{GMR}^{\mathrm{a}}(90 \% \mathrm{Cl})$} & \multirow[t]{2}{*}{$P$-value } \\
\hline & $\begin{array}{l}\text { Oseltamivir } \\
\text { alone }(n=19)\end{array}$ & $\begin{array}{l}\text { Oseltamivir + } \\
\text { dexamethasone }(n=19)\end{array}$ & & \\
\hline \multicolumn{5}{|l|}{ Metabolic ratio } \\
\hline Mean & 18.06 & 16.75 & $0.92(0.87,0.97)$ & 0.02 \\
\hline SD & 3.82 & 4.04 & & \\
\hline
\end{tabular}

Notes: The metabolic ratio was calculated as $\mathrm{AUC}_{0-48 \mathrm{~h}}$ (oseltamivir carboxylate)/AUC ${ }_{0-48 \mathrm{~h}}$ (oseltamivir). ${ }^{\mathrm{a}} \mathrm{Geometric}$ mean ratio $(90 \% \mathrm{Cl})$ of (oseltamivir + dexamethasone)/ (oseltamivir alone). ${ }^{\ddagger} P$-value was measured using a mixed-effect model in SAS software version 9.3 .

Abbreviations: GMR, geometric mean ratio; Cl, confidence interval; SD, standard deviation. 
expression of CES. Therefore, significant drug-drug interactions could occur when oseltamivir and dexamethasone are coadministered. This study investigated the influence of coadministering dexamethasone and oseltamivir on the PK of oseltamivir in healthy volunteers. The results showed that systemic exposure to oseltamivir carboxylate is slightly but significantly reduced after multiple administrations of dexamethasone, whereas systemic exposure to oseltamivir is relatively unchanged regardless of dexamethasone treatment.

PK drug-drug interactions involve absorption, distribution, metabolism, and excretion. Potential sites of interaction include the gastrointestinal tract, tissue-binding sites, drug-metabolizing enzymes, drug transporters, and renal excretion system. ${ }^{14}$ In the present study, oseltamivir and its active metabolite (oseltamivir carboxylate) reached their peak concentration $0.5-4.0$ and 2.0-6.0 h after administration of oseltamivir, respectively, both with and without dexamethasone coadministration. In addition, peak concentration of and systemic exposure to oseltamivir for both treatments (ie, oseltamivir alone or oseltamivir + dexamethasone) were similar. These results suggest that dexamethasone has little effect on oseltamivir absorption.

Several in vitro and in vivo studies have been conducted to determine the effects of dexamethasone on CES; however, conclusions from these studies are inconsistent. ${ }^{13,15-17}$ In the present study, we consider trends in the systemic exposure to oseltamivir carboxylate and the metabolic ratio after multiple administrations of dexamethasone, which was determined to be a CES inhibitor consistent with findings of previous studies. In a study of Quinney et al, ${ }^{18}$ dexamethasone was shown to be a very weak inhibitor of CES at high millimolar concentrations through inhibition of CES1- and CES2-catalyzed hydrolysis of 4-methylumbelliferyl acetate. In addition, Takahashi et $\mathrm{al}^{12}$ reported that dexamethasone weakly inhibits CES1-catalyzed imidaprilat formation from imidapril in human liver.

In contrast to systemic exposure, urinary levels and renal clearance of oseltamivir and oseltamivir carboxylate were increased, supporting the idea that dexamethasone affects the renal clearance of oseltamivir. Absorbed oseltamivir is primarily metabolized to oseltamivir carboxylate and then eliminated entirely by renal excretion. ${ }^{7}$ One possibility is that dexamethasone stimulates the clearance of oseltamivir carboxylate, leading to low plasma concentrations. A similar interaction has been proposed to occur when corticosteroids and salicylic acid are coadministered. ${ }^{19-21}$ However, an increased glomerular filtration rate and diminished tubular reabsorption of water after treatment with corticosteroids might partially explain their ability to increase the clearance of salicylate as well as the results observed for oseltamivir. $^{22}$

Although statistically significant, the $12 \%$ decrease in $\mathrm{AUC}_{0-48 \mathrm{~h}}$ observed for oseltamivir carboxylate is unlikely to be clinically relevant. Previous studies have shown that the approved adult treatment dose of $75 \mathrm{mg}$ twice daily maintains concentrations exceeding the half-maximal inhibitory concentration $\left(\mathrm{IC}_{50}\right)$ values for all tested influenza strains by at least 50 -fold. Further, the recommended dosage to maintain the steady-state plasma trough concentrations of oseltamivir carboxylate (the active metabolite) remains above the minimum inhibitory concentration for all influenza strains tested. ${ }^{7}$ From a clinical perspective, the potential of oseltamivir to have clinically relevant drug interactions during the treatment of influenza infections is low, and to date, there is no evidence in the literature of any significant treatment failures attributable to the concomitant use of oseltamivir and dexamethasone. Therefore, the observed decreases in $\mathrm{AUC}_{0-48 \mathrm{~h}}$ for oseltamivir carboxylate should have little effect on treatment outcomes, and a dose adjustment may not be necessary to produce the desired therapeutic effect in these cases.

The present study was conducted with low doses of dexamethasone. High-dose dexamethasone $(3-6 \mathrm{mg} / \mathrm{kg})$ treatments at the beginning of ARDS are an accepted therapeutic strategy. ${ }^{23}$ This study was designed based on the hypothesis that dexamethasone will act as an inducer or inhibitor of CES enzyme. The effect on CES by dexamethasone could be assessed through administering dexamethasone for sufficient days, even with a low dose. This study was not conducted in influenza or ARDS patients, and we proceeded to low doses of dexamethasone in consideration of the safety of the healthy volunteers; in this regard, dosage of oseltamivir has also not followed the standard treatment. Therefore, our results should be interpreted with caution. Further studies examining the interaction between oseltamivir and dexamethasone in influenza patients will be required to verify these results.

\section{Conclusion}

Coadministration of dexamethasone with oseltamivir slightly decreased systemic exposure to oseltamivir and oseltamivir carboxylate in healthy volunteers. These results suggest that CES1 is inhibited by coadministration of dexamethasone and oseltamivir in humans. However, coadministration of dexamethasone and oseltamivir had no clinically relevant effects on the PK of oseltamivir; based on these results, dexamethasone can be coadministered with oseltamivir. 


\section{Acknowledgments}

This study was supported by a research grant from the Basic Science Research Program through the National Research Foundation (NRF-2012R1A1A2000823), Republic of Korea, and by a grant of the Korea Health Technology R\&D Project through the Korea Health Industry Development Institute (KHIDI), funded by the Ministry of Health \& Welfare, Republic of Korea (HI14C2750). The abstract of this research was presented at the ASCPT 2016 Annual Meeting on March 8-12, 2016, in San Diego, CA, USA, and is published in the journal Clinical Pharmacology and Therapeutics (2016;99(Suppl 1):S5-S107).

\section{Author contributions}

All authors made substantial contributions to conception and design, acquisition of data, or analysis and interpretation of data, and drafting the article or revising it critically for important intellectual content, and gave final approval of the version to be published.

\section{Disclosure}

The authors declare no conflicts of interest in this work.

\section{References}

1. Hatfield MJ, Potter PM. Carboxylesterase inhibitors. Expert Opin Ther Pat. 2011;21(8):1159-1171.

2. Merali Z, Ross S, Pare G. The pharmacogenetics of carboxylesterases: CES1 and CES2 genetic variants and their clinical effect. Drug Metab Drug Interact. 2014;29(3):143-151.

3. Hosokawa M. Structure and catalytic properties of carboxylesterase isozymes involved in metabolic activation of prodrugs. Molecules. 2008;13(2):412-431.

4. Laizure SC, Herring V, Hu Z, Witbrodt K, Parker RB. The role of human carboxylesterases in drug metabolism: have we overlooked their importance? Pharmacotherapy. 2013;33(2):210-222.

5. Patrick KS, Straughn AB, Minhinnett RR, et al. Influence of ethanol and gender on methylphenidate pharmacokinetics and pharmacodynamics. Clin Pharmacol Ther. 2007;81(3):346-353.

6. Zhu HJ, Markowitz JS. Activation of the antiviral prodrug oseltamivir is impaired by two newly identified carboxylesterase 1 variants. Drug Metab Dispos. 2009;37(2):264-267.

7. He G, Massarella J, Ward P. Clinical pharmacokinetics of the prodrug oseltamivir and its active metabolite Ro 64-0802. Clin Pharmacokinet. 1999;37(6):471-484.
8. Davies BE. Pharmacokinetics of oseltamivir: an oral antiviral for the treatment and prophylaxis of influenza in diverse populations. $J$ Antimicrob Chemother. 2010;65(Suppl 2):ii5-ii10.

9. Dutkowski R, Thakrar B, Froehlich E, Suter P, Oo C, Ward P. Safety and pharmacology of oseltamivir in clinical use. Drug Saf. 2003; 26(11):787-801.

10. McClellan K, Perry CM. Oseltamivir: a review of its use in influenza. Drugs. 2001;61(2):263-283.

11. Xu T, Qiao J, Zhao L, et al. Effect of dexamethasone on acute respiratory distress syndrome induced by the $\mathrm{H} 5 \mathrm{~N} 1$ virus in mice. Eur Respir $J$. 2009;33(4):852-860.

12. Takahashi S, Katoh M, Saitoh T, Nakajima M, Yokoi T. Different inhibitory effects in rat and human carboxylesterases. Drug Metab Dispos. 2009;37(5):956-961.

13. Zhu W, Song L, Zhang H, Matoney L, LeCluyse E, Yan B. Dexamethasone differentially regulates expression of carboxylesterase genes in humans and rats. Drug Metab Dispos. 2000;28(2):186-191.

14. Anglicheau D, Flamant M, Schlageter MH, et al. Pharmacokinetic interaction between corticosteroids and tacrolimus after renal transplantation. Nephrol Dial Transpl. 2003;18(11):2409-2414.

15. Shi D, Yang J, Yang D, Yan B. Dexamethasone suppresses the expression of multiple rat carboxylesterases through transcriptional repression: evidence for an involvement of the glucocorticoid receptor. Toxicology. 2008;254(1-2):97-105.

16. Furihata T, Hosokawa M, Fujii A, Derbel M, Satoh T, Chiba K Dexamethasone-induced methylprednisolone hemisuccinate hydrolase: its identification as a member of the rat carboxylesterase 2 family and its unique existence in plasma. Biochem Pharmacol. 2005;69(8): 1287-1297.

17. Hattori K, Igarashi M, Itoh M, Tomisawa H, Ozawa N, Tateishi M Purification and characterization of glucocorticoid-inducible steroid esterase in rat hepatic microsomes. Biochem Pharmacol. 1992;43(9): 1929-1937.

18. Quinney SK, Sanghani SP, Davis WI, et al. Hydrolysis of capecitabine to 5'-deoxy-5-fluorocytidine by human carboxylesterases and inhibition by loperamide. J Pharmacol Exp Ther. 2005;313(3):1011-1016.

19. Miners JO. Drug interactions involving aspirin (acetylsalicylic acid) and salicylic acid. Clin Pharmacokinet. 1989;17(5):327-344.

20. Miners JO, Grgurinovich N, Whitehead AG, Robson RA, Birkett DJ. Influence of gender and oral contraceptive steroids on the metabolism of salicylic acid and acetylsalicylic acid. Br J Clin Pharmacol. 1986; 22(2):135-142.

21. Verbeeck RK. Pharmacokinetic drug interactions with nonsteroidal anti-inflammatory drugs. Clin Pharmacokinet. 1990;19(1):44-66.

22. Klinenberg JR, Miller F. Effect of corticosteroids on blood salicylate concentration. JAMA. 1965;194(6):601-604.

23. Ariani F, Liu K, Jing Z, Qu J. Glucocorticosteroid in treatment of severe pneumonia. Mediators Inflamm. 2013;2013:865635.

\section{Publish your work in this journal}

Drug Design, Development and Therapy is an international, peerreviewed open-access journal that spans the spectrum of drug design and development through to clinical applications. Clinical outcomes, patient safety, and programs for the development and effective, safe, and sustained use of medicines are the features of the journal, which

\section{Dovepress}

has also been accepted for indexing on PubMed Central. The manuscript management system is completely online and includes a very quick and fair peer-review system, which is all easy to use. Visit http://www.dovepress.com/testimonials.php to read real quotes from published authors. 